\title{
Clinical Course of High-Frequency Millimeter-Wave (162 GHz) Induced Ocular Injuries and Investigation of Damage Thresholds
}

\author{
Masami Kojima ${ }^{1,2,3}$ (D) Yukihisa Suzuki ${ }^{4}$. Takafumi Tasaki ${ }^{5,6} \cdot$ Yoshinori Tatematsu $^{7}$. \\ Maya Mizuno ${ }^{8} \cdot$ Masafumi Fukunari $^{7} \cdot$ Hiroshi Sasaki $^{1,2}$
}

Received: 4 December 2019 / Accepted: 1 June 2020/Published online: 11 June 2020

(C) The Author(s) 2020

\begin{abstract}
The objective was to investigate the clinical course of ocular damage and the healing process in eyes exposed to $162 \mathrm{GHz}$ millimeter wave (MMW). As a 162-GHz electromagnetic wave source, a gyrotron FU CW GV was used. An ocular damaged rabbit model with good reproducibility for $162 \mathrm{GHz}$ MMW exposure was developed, and damage occurrence threshold values were obtained. The right eyes of pigmented rabbits were exposed to $162 \mathrm{GHz}$ from a spot-focus-type lens antenna, and the non-exposed contralateral eyes were used as control eyes. Slit-lamp examination 1 day after MMW exposure revealed a round area of opacity, characterized by fluorescein staining indicating damaged epithelial cells in the central pupillary zone. Corneal edema, indicative of corneal stromal damage, peaked 2-3 days after exposure, with thickness gradually subsiding to normal by 9 days after exposure. Power densities of $162 \mathrm{GHz} \mathrm{MMW}$ causing ocular damage with probabilities of 10, 50, and $90 \%$ obtained by probit analysis were 173,252 , and $368 \mathrm{~mW} / \mathrm{cm}^{2}$, respectively.
\end{abstract}

Keywords Millimeter wave $\cdot$ Pigmented rabbit eye $\cdot$ Corneal epithelial damage $\cdot$ Ocular damage dose $\cdot$ Gyrotron

\section{Introduction}

Electromagnetic waves at millimeter $(30-300 \mathrm{GHz}, \mathrm{MMW})$ and submillimeter ( $300 \mathrm{GHz}-$ $3 \mathrm{THz}$ ) waveband frequencies are expected to be increasingly used in advanced technologies such as high-speed and broadband communications, wireless power transmission, remote sensing, and more. However, at present, there is sparse in vivo research data regarding the impact of these energy forms on living tissues.

Masami Kojima

m-kojima@kanazawa-med.ac.jp

Extended author information available on the last page of the article 
It was reported several decades ago that energy in the frequency range 10-300 $\mathrm{GHz}$ was absorbed by the skin surface and the surface tissue of eyeballs [1]. Studies have examined the effects to eyes of MMW exposure [2-8], but because their exposure methodology (especially, shape of antenna (radiation pattern of antenna) for MMW delivery) and experimental animals differed, the results of these studies cannot be directly compared. We have previously reported data on the threshold of eye injury in Dutch rabbits exposed to 40, 75, and $95 \mathrm{GHz} \mathrm{MMW}$ using uniform experimental procedures [7].

One aim of the present study was to compare the threshold of eye injury for $162 \mathrm{GHz}$ MMW exposure in the same rabbit breed using the same experimental method as in our previous reports. A high-power MMW exposure device is essential to create a highly reproducible ocular injury model. However, in general, it is technologically difficult to realize high-power MMW exposure by solid-state semiconductors, which we have used in our previous study, approximately from $100 \mathrm{GHz}$ to $10 \mathrm{THz}$. This is a well-known problem termed "THz gap" [9]. Therefore, we focused on the gyrotron as a candidate high-power MMW wave source above $100 \mathrm{GHz}$.

The gyrotron is an oscillating tube to which the cyclotron resonance maser principle is applied utilizing the mass change of electrons due to the relativistic effect. Applications of the gyrotron are being developed including electronic heating in the plasma science and fusion fields, the output of which reaches $1 \mathrm{MW}$ (Mega Watt) at a frequency of about $100 \mathrm{GHz}$ [10]. As the University of Fukui was undertaking research using a gyrotron and was looking for joint researchers, we considered the possibility of using a gyrotron for the wave source in the present study. Thus, a second aim was to use a gyrotron to investigate the threshold of ocular damage associated with exposure to $162 \mathrm{GHz}$ MMW. A third aim was to clarify the clinical course of ocular damage and its healing process after $162 \mathrm{GHz}$ MMW exposure.

As far as the authors know, there are no reports of millimeter-wave eye injuries above $100 \mathrm{GHz}$ other than one by Rosenthal et al. who investigated the effects of exposure to $107 \mathrm{GHz}$ [2]. This is the first report of eye injuries caused by exposure to $162 \mathrm{GHz} \mathrm{MMW}$.

\section{Materials and Methods}

\subsection{Exposure System}

Figure 1 shows a block diagram of the exposure system used in this study. A 162-GHz MMW energy source in continuous wave mode was generated by the gyrotron FU CW GV constructed in University of Fukui [11]. A lens antenna (diameter $100 \mathrm{~mm}$ ) with a 200-mm focal point and 6.5-mm FWHM (full width at half its maximum intensity) beam width was used to deliver MMW to rabbit cornea. The power distribution was verified using a waveguide probe (AOEWGP-02/3.0, Elmika, Vilnius, Lithuania) with gain obtained by the three-antenna method [12] and a power meter (Erikson PM4, Virginia Diodes, Inc., USA), and total power incident to the lens antenna was monitored using a power meter (Erikson PM5, Virginia Diodes, Inc., USA).

Figure 2 shows the method of measuring spatial power distribution. The measurement plane was $200 \mathrm{~mm}$ from the lens antenna, which is the focal point of the antenna, and perpendicular to the z-axis. The open-ended waveguide probe was swept across this plane from (-xmax, - ymax) to (xmax, ymax). The intervals of measurement points, i.e., $\Delta x$ and $\Delta y$, were $0.5 \mathrm{~mm}$. The results of measurement of power distribution at the focal point are shown in 


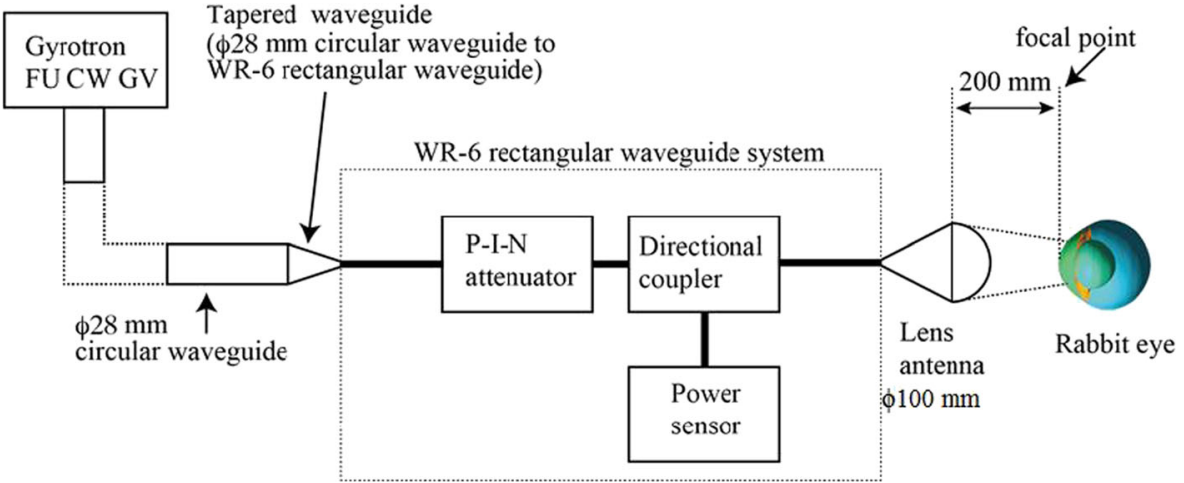

Fig. 1 A block diagram of the developed $162 \mathrm{GHz}$ exposure system

Fig. 3. In this figure, the vertical axis is normalized by the maximum value at the axis of the lens antenna. FWHM was estimated by this result as $6.5 \mathrm{~mm}$.

In this study, the spatially averaged power per area with a diameter of $13 \mathrm{~mm}$, which is the average size of the corneal region in Dutch-belted rabbits, was calculated from the monitored power and measurement results of the power distribution at the focal plane. The relationship between input power to the antenna and averaged power density at the focal plane was determined by measurement of lens antenna properties including power loss by the antenna and the coupler.

\subsection{Experimental Animals}

The animals were managed in accordance with practices and experimental protocols reviewed and approved by the Animal Research Committee, University of Fukui. All animal

\section{Lens antenna}

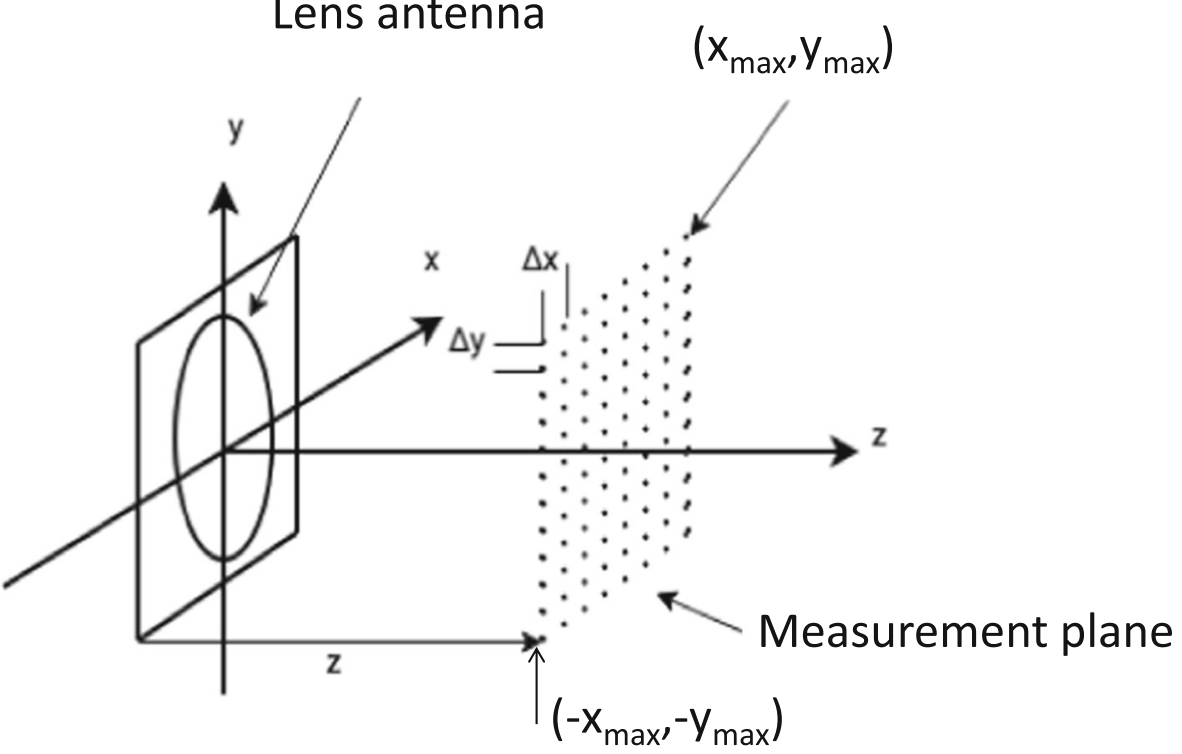

Fig. 2 Method of measuring spatial power distribution 
$-3 d B$

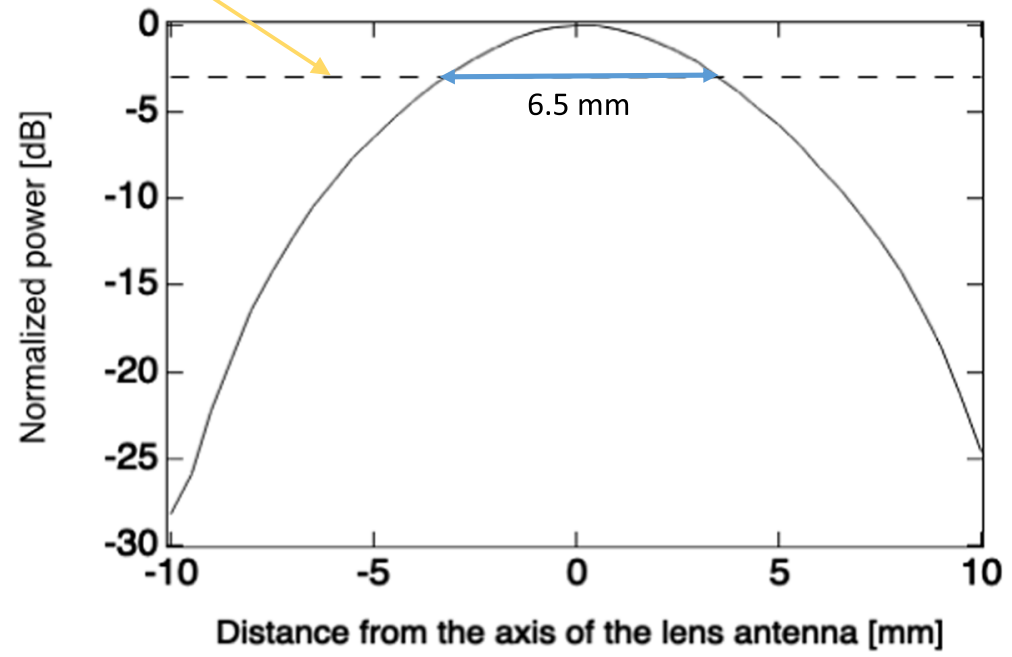

Fig. 3 Measurement result of power distribution at the focal point

experiments were conducted in accordance with the animal experimentation guidelines of Kanazawa Medical University (Kahoku, Japan), University of Fukui (Fukui, Japan), and the ARVO Statement for the Use of Animals in Ophthalmic and Vision Research (ARVO, 2016) [13].

Forty-six male Dutch-belted pigmented rabbits (age: 10-13 weeks, mean \pm SD weight: 1.85 $\pm 0.03 \mathrm{~kg}$ ) were purchased from Sankyo Labo Service Co., Inc. (Toyama, Japan). They were housed individually in stainless steel rabbit housing cages kept in a cargo van as short-term animal housing. Before starting this experiment, we confirmed that the cargo van animal housing did not differ from a conventional animal facility, at least for short-term housing [14].

Each rabbit was injected subcutaneously or intramuscularly (IM) with a solution containing 0.25- $0.50 \mathrm{mg} / \mathrm{kg}$ of medetomidine hydrochloride (Domitor, Nippon Zenyaku Kogyo Co., Ltd., Fukushima, Japan) and 0.025-0.050 $\mathrm{mg} / \mathrm{kg}$ buprenorphine hydrochloride (Buprenorphine Inj. 0.2 mg "NISSIN", Nissin Pharmaceutical Co., Ltd., Yamagata, Japan) to induce general anesthesia or to provide sedation and immobilized in an acrylic rabbit restrainer which was specially constructed for studies of exposure to MMW [5]. All rabbit eyes were pre-examined using a slit-lamp microscope (SL-130, Zeiss, Tokyo, Japan) to ensure absence of abnormalities, and each eye was photographed to confirm the pre-experimental ocular condition. Immediately before MMW exposure, 2\% lidocaine hydrochloride topical anesthetic (Xylocaine 2\%, AstraZeneca, Osaka, Japan) was instilled to each eye. The upper and lower eyelids of both eyes (exposed and control) were held open with tape. As necessary, to prevent corneal epithelial damage from corneal desiccation, and to ensure equivalent ocular surface conditions among rabbits, saline drops were instilled to the experimental eyes before exposure and the contralateral eyes as controls. All rabbits were administered topical ofloxacin eye drops and ointment (Tarivid, Santen Pharmaceutical Co., Ltd., Osaka, Japan) to prevent secondary infection after experimental treatment was competed each day. Temperature and humidity during exposure were maintained at $24 \pm 2{ }^{\circ} \mathrm{C}$ and $60 \pm 10 \%$, respectively, using an air conditioner and dehumidifier. Anesthesia was reversed with $0.8-$ 
$1.5 \mathrm{mg} / \mathrm{kg}$ IM atipamezole hydrochloride (Antisedan, Nippon Zenyaku Kogyo Co., Ltd., Fukushima, Japan) to aid rabbit recovery.

\subsection{MMW Exposure}

Prior to the animal experiment, the most uniformly irradiated site was identified by investigation of the detailed beam profile of the antenna used in this experiment. The center of the corneal surface of the right eye was positioned $200 \mathrm{~mm}$ from the antenna aperture and on the line of maximum radiation of the antenna. According to Kojima's methodology $[5,6]$, the exposure point was set using red and green laser pointers on a target, and 46 right eyes of Dutch rabbits were continuously exposed to $162 \mathrm{GHz} \mathrm{MMW}$ at $60-600 \mathrm{~mW} / \mathrm{cm}^{2}$ for $6 \mathrm{~min}$. The corresponding 46 left eyes were unexposed and regarded as control eyes.

\subsection{Corneal Surface Temperature}

Corneal surface temperature was recorded in exposed eyes at $5 \mathrm{~s}$ prior to exposure and $5 \mathrm{~s}$ before the end of exposure using a thermography camera (FLIR T620, FLIR Systems, Inc., Oregon, USA).

\subsection{Examination of Ocular Injury}

The anterior segment was evaluated before exposure, and at $10 \mathrm{~min}, 1,2,3$, 6, and 9 days after exposure. Corneal epithelial damage was observed by slit-lamp microscopy using a modified Rosenthal's method [2] where fluorescein staining revealed damaged corneal epithelial cells [7]. Corneal cross-sectional thickness was measured and recorded by optical coherence tomography (CIRRIS HD-OCT plus model 5000, Zeiss, Tokyo, Japan), and corneal opacity was assessed by slit-lamp microscopy. Measurements of corneal thickness were taken at three points, $1.5 \mathrm{~mm}$ above (upper) and $1.5 \mathrm{~mm}$ below (lower) the pupillary central region, and at the center of the pupil (middle).

\subsection{Categorization of Corneal Epithelial Injury}

Corneal epithelial damage was defined as presence of fluorescein staining, indicating an area of epithelial injury in the central pupillary zone of the exposed eye (right eye), with no similar injury observed in its corresponding unexposed eye (left eye). Other types of corneal epithelial damage, such as desiccation of the cornea (i.e., dry eye) and mechanical damage, were excluded and in any case did not occur.

\subsection{Data Analysis}

The probability of corneal damage at 1 day after exposure to $162 \mathrm{GHz}$ MMW at different power densities was evaluated by maximum likelihood estimation (MLE) using probit analysis [15]. The morphological changes in the cornea assessed by slit-lamp microscopy, including corneal opacity, and fluorescein staining revealing corneal epithelial damage, and anterior segment optical coherence tomography (ASOCT) to measure corneal edema at 1 day after exposure, were used for the probit analyses. The dose-response relationship between corneal injury and power density was evaluated by fitting with a cumulative lognormal distribution 
function for probit analysis using R Ver 3.5.2 software [16]. The MMW power density indicating the probability of ocular damage was defined as damage dose (DD) and was derived from the best-fit probit function with $p \leq 0.01$.

\section{Results and Discussion}

\subsection{Exposure to $162 \mathrm{GHz}$ at $600 \mathrm{~mW} / \mathrm{cm}^{2}$ for $6 \mathrm{Min}$}

Since this was the first investigation of ocular injury using a gyrotron, examination of characteristics of MMW induced eye damage required a sufficiently high exposure dose. Two right eyes were exposed to $162 \mathrm{GHz}$ at $600 \mathrm{~mW} / \mathrm{cm}^{2}$ for $6 \mathrm{~min}$. Our ethical protocol for discontinuation was a cornea becoming turbid during exposure or the animal showing a strong rejection response.

Experiments were performed using two rabbits in which responses requiring discontinuation did not occur during 6-min exposure. At 10 min after exposure, miosis was observed in both animals indicating induced ocular inflammation. At 1 day after exposure, in both rabbits, the anterior segment including the cornea was completely opaque obscuring observation of the pupil shape. In both rabbits, the unexposed control eyes (left eye) remained normal throughout the experimental period.

The effect on the rabbit eye by exposure to $162 \mathrm{GHz}$ at $600 \mathrm{~mW} / \mathrm{cm}^{2}$ for $6 \mathrm{~min}$ was similar to damage caused by high dose exposure to 40, 75, and $95 \mathrm{GHz}$ reported by Kojima et al [7]. Ocular damage due to high-intensity MMW exposure is miosis and corneal epithelial damage (fluorescein staining findings) observed immediately (10 min) after exposure [7]. Corneal opacification occurs to the extent that the pupil cannot be observed at 1 day after exposure.

Experiments including exposure to $162 \mathrm{GHz}$ at doses above $600 \mathrm{~mW} / \mathrm{cm}^{2}$ or at this level for more than 6 min were deemed unethical and not conducted.

\subsection{GHz MMW Ocular Damage Model}

Figure 4 shows a representative example of eye injury caused by $162 \mathrm{GHz}$ at $480 \mathrm{~mW} / \mathrm{cm}^{2}$ for 6 min exposure.

Thermographic measurement of corneal surface temperature showed $31.5^{\circ} \mathrm{C}$ before exposure and $48.5^{\circ} \mathrm{C}\left(\Delta T=17.0^{\circ} \mathrm{C}\right) 5 \mathrm{~s}$ before the end of exposure.

Slit-lamp examination at $10 \mathrm{~min}$ after exposure $\left(162 \mathrm{GHz} 480 \mathrm{~mW} / \mathrm{cm}^{2}\right.$ for $6 \mathrm{~min}$ ) revealed corneal epithelial damage (fluorescein staining findings) in 2 of 10 eyes. And in all 10, including those without corneal epithelial damage, ASOCT revealed cornea thicknesses was reduced following exposure. Before exposure, the corneal thicknesses (upper, middle, lower) measured by ASOCT showed normal values of $0.36,0.36$, and $0.37 \mathrm{~mm}$, respectively. In the observation at $10 \mathrm{~min}$ after exposure, those were thinned to 0.33 , 0.32 , and $0.30 \mathrm{~mm}$, respectively. Unexposed control eyes (left eye) remained normal throughout the experimental period.

Kojima et al. reported the initial steps of corneal injury at 10 min after exposure to MMW $(40,75,95 \mathrm{GHz})$ are "thinning of cornea (corneal desiccation)" and "partial dropout of corneal epithelial cells". In the present study, thinning of the cornea was observed in all 10 cases, but fluorescein staining was observed in only 2 of 10 cases. In the fluorescein staining test at 1 day after exposure, all 10 eyes were positive and showed corneal opacity, so it is considered that 


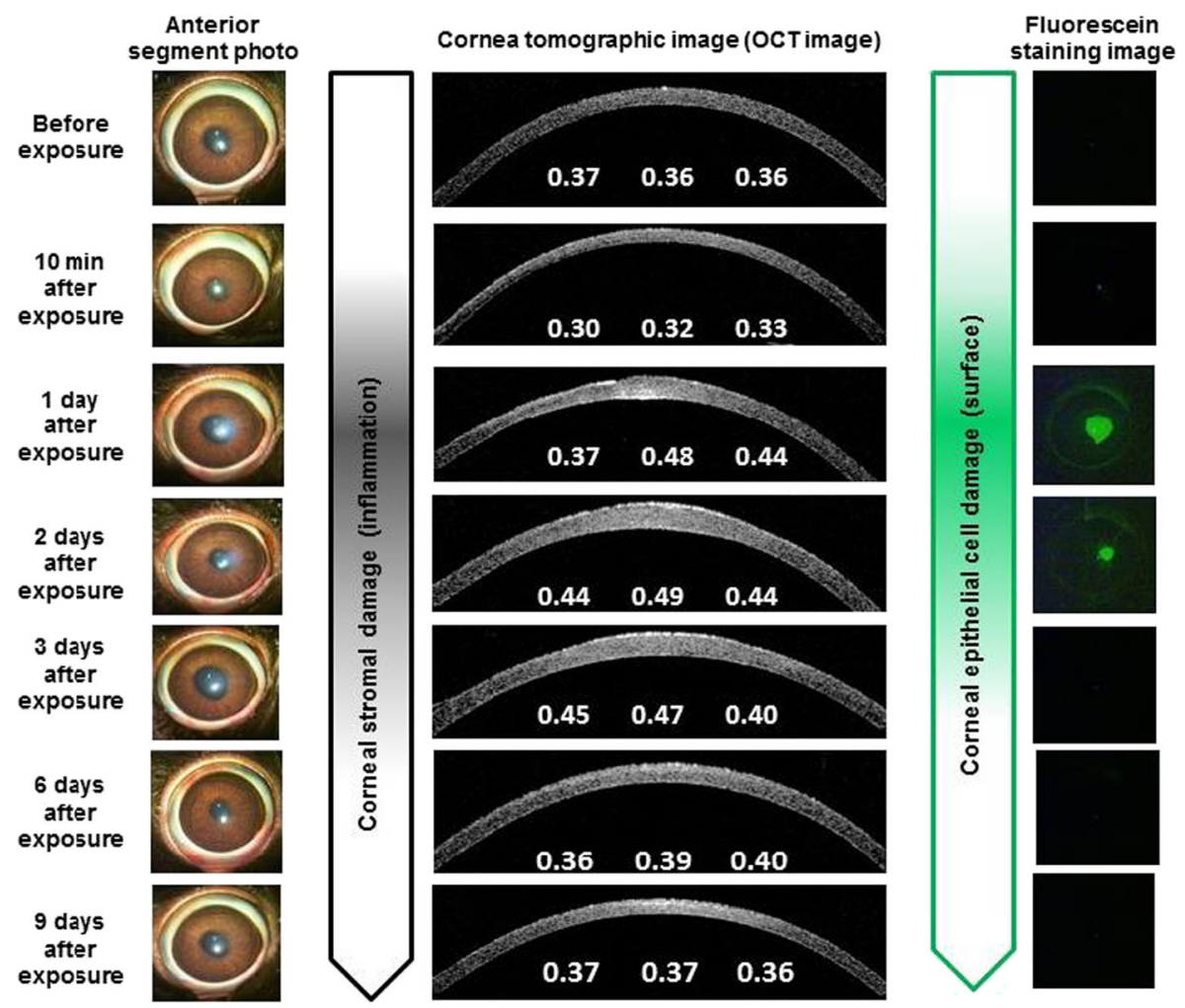

Fig. 4 Representative examples of eyes with corneal damage and their clinical courses following their exposure to $162 \mathrm{GHz} \mathrm{MMW}$ at $480 \mathrm{~mW} / \mathrm{cm}^{2}$ for $6 \mathrm{~min}$. The numbers indicate corneal thickness in millimeters

the exposure dose was sufficient to cause ocular damage. The cause of the discrepancy between the findings at $10 \mathrm{~min}$ and at 1 day after exposure is unknown at present, but examination at $10 \mathrm{~min}$ after exposure can be said to be unsuitable for determining ocular damage due to $162 \mathrm{GHz}$ MMW exposure.

In the present study, corneal opacity in exposed eyes at 1 day after exposure was observed in the central pupillary zone, and fluorescein staining was present where corneal opacity was observed. In the measurement of corneal thickness by ASOCT at 1 day after exposure, edema was observed in the upper $(0.44 \mathrm{~mm})$ and central cornea $(0.48 \mathrm{~mm})$.

Slit-lamp microscopy at 2 days after exposure revealed the degree of corneal turbidity was reduced compared to that at 1 day after exposure. In addition, the area of fluorescein staining at 2 days after exposure was smaller than that at 1 day after exposure. These findings suggest that ocular damage caused by exposure to $162 \mathrm{GHz}$ at $480 \mathrm{~mW} / \mathrm{cm}^{2}$ for 6 min peaked around 1 day after exposure, and healing gradually started. On the contrary, in the examination of corneal thickness at 2 days after exposure, edema was observed to have increased.

Three days after exposure, corneal opacity recovered and fluorescein staining disappeared. However, corneal edema was somewhat improved but still present. Corneal edema was observed at 6 days after exposure, but returned to normal corneal thickness by 9 days after exposure. Unexposed control eyes (left eye) remained normal throughout the experimental period. 
Ocular damage by exposure to $162 \mathrm{GHz}$ at $480 \mathrm{~mW} / \mathrm{cm}^{2}$ for 6 min was judged to be appropriate as a rabbit model eye for MMW exposure, as the degree of damage was ethically acceptable. A total of 10 rabbits were similarly exposed over different days to investigate the reproducibility of this MMW exposure animal model. The average corneal surface temperature was $48.5 \pm 1.2{ }^{\circ} \mathrm{C}\left(\mathrm{CV}=2.5 \%\right.$, max temperature: $51.2^{\circ} \mathrm{C}$, Min temperature $\left.46.7^{\circ} \mathrm{C}\right)$.

Ocular damage caused by exposure to $162 \mathrm{GHz}$ at $480 \mathrm{~mW} / \mathrm{cm}^{2}$ for $6 \mathrm{~min}$ has the following 3 characteristics: (1) Not severe enough to cause the rabbit cornea to become opaque during exposure. (2) Ocular damage after exposure is reversible turbidity that reaches its peak around 1 day after exposure and gradually recovers. (3) Ocular damage caused by this exposure, including corneal opacity, corneal epithelial injury (defined by fluorescein staining), and corneal edema, show good simultaneous and day-to-day reproducibility (10 rabbits similarly exposed showed similar ocular damage with little variation). Accordingly, we determined that $162 \mathrm{GHz}$ at $480 \mathrm{~mW} / \mathrm{cm}^{2} 6$-min exposure condition could provide a model for ocular damage of $162 \mathrm{GHz}$ MMW.

\subsection{Threshold of $162 \mathrm{GHz}$ MMW Ocular Damage Model}

Table 1 shows the relationship between different power density values of $162 \mathrm{GHz}$ (incident power density) and the proportion of eyes damaged (damaged/exposed). The corneal surface temperature measured during MMW exposure ( $5 \mathrm{~s}$ before the end of exposure) is also shown. Under the exposure condition of $162 \mathrm{GHz}$ at $480 \mathrm{~mW} / \mathrm{cm}^{2}$ for $6 \mathrm{~min}$, the corneal surface temperature was $48.5 \pm 1.2{ }^{\circ} \mathrm{C}$, and corneal epithelial injury (positive fluorescein staining),

Table 1 Summary of the results at 1 day after exposure of rabbit eyes to $162 \mathrm{GHz} \mathrm{MMW}$

\begin{tabular}{|c|c|c|c|c|}
\hline $\begin{array}{c}162 \mathrm{GHZ} \\
1 \text { day after } \\
\text { exposure }\end{array}$ & $\begin{array}{c}\text { Corneal surface } \\
\text { temperature }\left({ }^{\circ} \mathrm{C}\right)^{*}\end{array}$ & $\begin{array}{c}\text { Corneal epithelium } \\
\text { damage }\end{array}$ & Corneal opaque & Corneal edema \\
\hline $600 \mathrm{~mW} / \mathrm{cm}^{2}{ }^{2} 6 \mathrm{~min}$ & 51.2 & Damage $+(2 / 2)$ & Damage $+(2 / 2)$ & Damage $+(2 / 2)$ \\
\hline $480 \mathrm{~mW} / \mathrm{cm}^{2}{ }^{2} 6 \mathrm{~min}$ & $48.5 \pm 1.2$ & Damage $+(10 / 10)$ & Damage $+(10 / 10)$ & Damage $+(10 / 10)$ \\
\hline $360 \mathrm{~mW} / \mathrm{cm}^{2} \_6 \mathrm{~min}$ & $45.5 \pm 1.0$ & Damage+ (6/8) & Damage $+(4 / 8)$ & Damage + (4/8) \\
\hline $330 \mathrm{~mW} / \mathrm{cm}^{2} \_6 \mathrm{~min}$ & $45.1 \pm 0.5$ & Damage+ $(3 / 3)$ & Damage $+(2 / 3)$ & Damage $+(3 / 3)$ \\
\hline $300 \mathrm{~mW} / \mathrm{cm}^{2}{ }^{2} 6 \mathrm{~min}$ & $44.4 \pm 0.8$ & Damage+ $(4 / 5)$ & Damage $+(2 / 5)$ & Damage $+(4 / 5)$ \\
\hline $240 \mathrm{~mW} / \mathrm{cm}^{2} \_6 \mathrm{~min}$ & $42.9 \pm 1.8$ & Damage+ (4/9) & Damage $+(1 / 9)$ & Damage $+(4 / 9)$ \\
\hline $120 \mathrm{~mW} / \mathrm{cm}^{2} \_6 \mathrm{~min}$ & $37.6 \pm 0.7$ & No damage $(0 / 6)$ & No damage $(0 / 6)$ & No damage $(0 / 6)$ \\
\hline $60 \mathrm{~mW} / \mathrm{cm}^{2}{ }^{2} 6 \mathrm{~min}$ & $34.3 \pm 0.8$ & No damage $(0 / 3)$ & No damage $(0 / 3)$ & No damage $(0 / 3)$ \\
\hline
\end{tabular}

* Corneal surface temperature was measured at $5 \mathrm{~s}$ before the end of exposure. Damage $+(\mathrm{n} / \mathrm{N})$ indicates damage was observed in $\mathrm{n}$ of $\mathrm{N}$ exposed eyes 


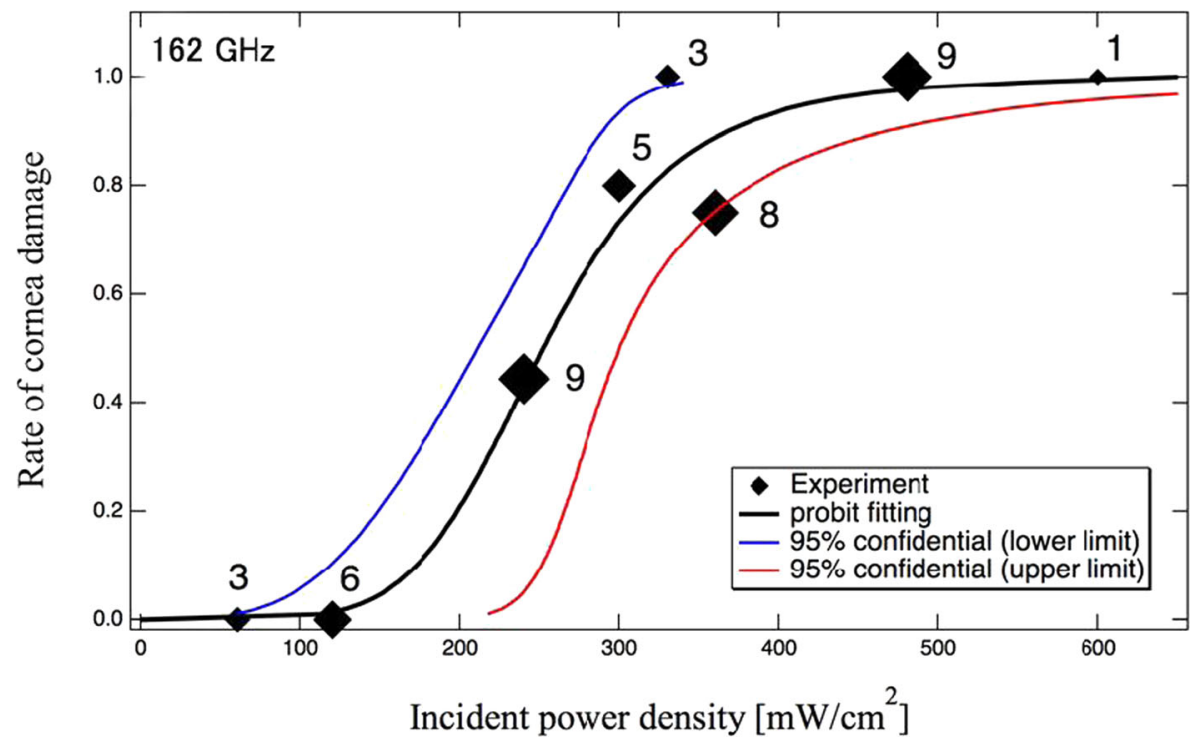

Fig. 5 Results of MLE with probit analysis for exposure to $162 \mathrm{GHz}$ MMW. The markers in the graph denote the dependence of the proportion of damaged eyes relative to the number of eyes exposed to the indicated power density (dose). The number of rabbit eyes at each power density correspond to the size of the diamond-shaped markers. The blue and red lines indicate the lower and upper limits of $95 \%$ confidence intervals, respectively

corneal opacification (slit-lamp observation), and corneal edema (ASOCT examination) were observed in all of 10 exposed eyes.

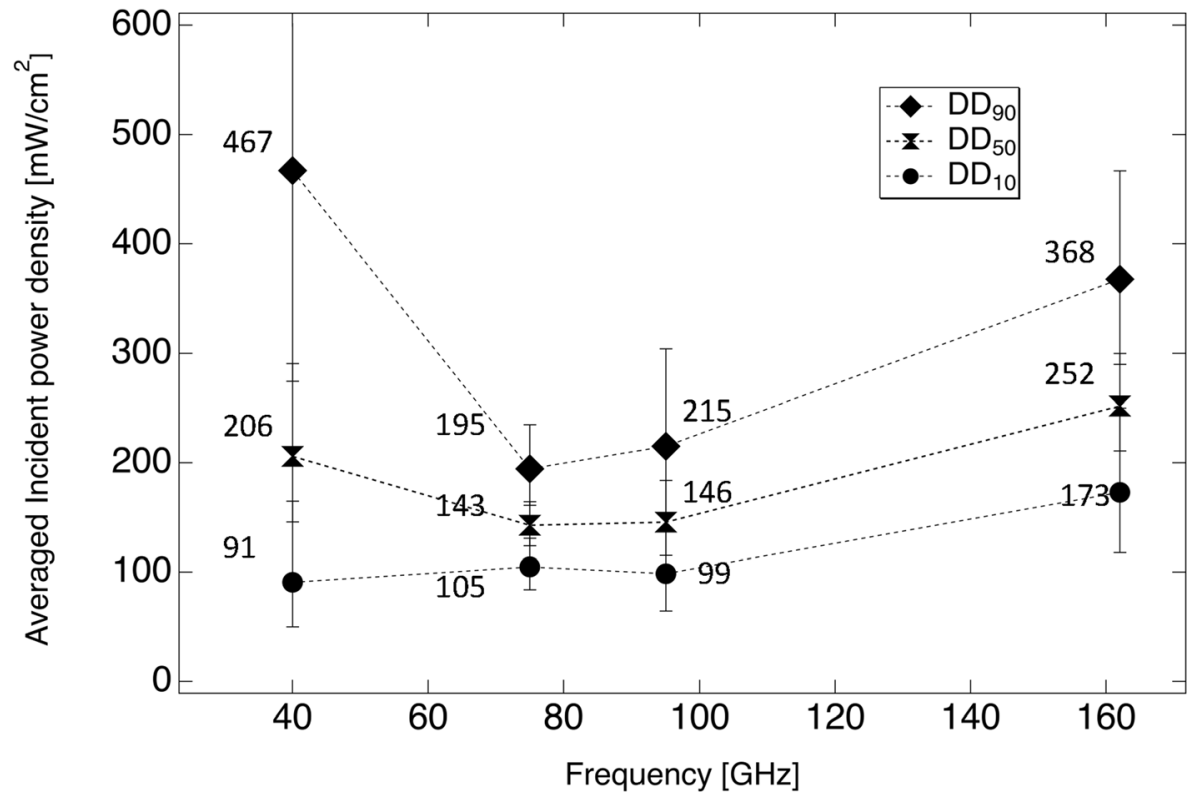

Fig. 6 Estimation of power density threshold level for 6 min exposure with probit analysis from 40 to $162 \mathrm{GHz}$. DD $10 \%\left(\mathrm{DD}_{10}\right), \mathrm{DD} 50 \%\left(\mathrm{DD}_{50}\right)$, and DD $90 \%\left(\mathrm{DD}_{90}\right)$ estimated from results of MLE for exposure to 40,75 , 95 [7], and $162 \mathrm{GHz}$ MMW. Error bars indicate 95\% confidence intervals for each marker 
Under the condition of $300 \mathrm{~mW} / \mathrm{cm}^{2}$ exposure for $6 \mathrm{~min}$, the corneal surface temperature was $44.4 \pm 0.8^{\circ} \mathrm{C}$, corneal epithelial damage and corneal edema were present in 4 of 5 exposed eyes, and corneal opacity in 2 of 5 .

A previous study compared the effects of exposure to 40,75 , and $95 \mathrm{GHz}$ MMW at $300 \mathrm{~mW} / \mathrm{cm}^{2}$ for 6 min using rabbit corneal surface temperature as an index and reported that the corneal surface temperature at $5 \mathrm{~s}$ before the end of exposure was in the order of $75(50.7 \pm$ $\left.1.9^{\circ} \mathrm{C}\right)>95\left(46.5 \pm 1.3{ }^{\circ} \mathrm{C}\right)>40 \mathrm{GHz}\left(41.3 \pm 1.3{ }^{\circ} \mathrm{C}\right)$ [7]. Since the protocols of the present experiment were the same as those of the previous experiment, except for the MMW source, results of the present experiment may reliably be compared with those of the previous. The corneal surface temperature at $5 \mathrm{~s}$ before the end of exposure to $162 \mathrm{GHz}$ at $300 \mathrm{~mW} / \mathrm{cm}^{2}$ for 6 min was $44.4 \pm 0.8{ }^{\circ} \mathrm{C}$. Differences in corneal surface temperature induced by exposure to $300 \mathrm{~mW} / \mathrm{cm}^{2}$ at different MMW frequencies seem to be due to penetration depth [17] depending on MMW frequency.

When exposed to $162 \mathrm{GHz}$ at $120 \mathrm{~mW} / \mathrm{cm}^{2}$ for $6 \mathrm{~min}$, the corneal surface temperature at $5 \mathrm{~s}$ before the end of exposure was $37.6 \pm 0.7{ }^{\circ} \mathrm{C}$, which is within the range of rabbit body temperature, $37.3-39.8{ }^{\circ} \mathrm{C}$ [18]. All 6 rabbits exposed to $162 \mathrm{GHz}$ at $120 \mathrm{~mW} / \mathrm{cm}^{2}$ for 6 min showed no ocular damage.

Under the condition of $162 \mathrm{GHz}$ at $60 \mathrm{~mW} / \mathrm{cm}^{2}$ for $6 \mathrm{~min}$, the cornea temperature at $5 \mathrm{~s}$ before the end of exposure was $34.3 \pm 0.8{ }^{\circ} \mathrm{C}$, which is below the rabbit average body temperature.

Based on the above, it was estimated that the onset threshold of ocular damage caused by exposure to $162 \mathrm{GHz}$ MMW for $6 \mathrm{~min}$ was in the range of $120-240 \mathrm{~mW} / \mathrm{cm}^{2}$.

We used the ocular damage model $\left(162 \mathrm{GHz}\right.$ at $480 \mathrm{~mW} / \mathrm{cm}^{2}$ for $6 \mathrm{~min}$ ) as a positive control, and in the process of reducing the power density of exposure, we defined the time point when the onset of ocular damage disappeared as a threshold, and performed ocular damage threshold research.

The relationship between ocular damage and $162 \mathrm{GHz}$ MMW at various power densities was evaluated by determining the probability of corneal damage at 1 day after exposure by MLE using probit analysis (Fig. 5). The black line plotted in the graph denotes the probability of ocular damage by power density (dose).

Figure 6 shows the results of probit analysis of 40,75, and $95 \mathrm{GHz}$ summed with $162 \mathrm{GHz}$ in the present study. The DD 10\% ( $\left.\mathrm{DD}_{10}\right)$, $\mathrm{DD} 50 \%\left(\mathrm{DD}_{50}\right)$, and $\mathrm{DD} 90 \%\left(\mathrm{DD}_{90}\right)$ was determined by MLE, following exposure to 40, 75, 95 (data from [7]), and $162 \mathrm{GHz}$ MMW. The $50 \%$ probability of ocular damage $\left(\mathrm{DD}_{50}\right)$ was in the order $162\left(252 \mathrm{~mW} / \mathrm{cm}^{2}\right)$ $>40\left(206 \mathrm{~mW} / \mathrm{cm}^{2}\right)>95\left(146 \mathrm{~mW} / \mathrm{cm}^{2}\right)>75 \mathrm{GHz}\left(143 \mathrm{~mW} / \mathrm{cm}^{2}\right)$.

\section{Conclusion}

We developed a 162-GHz MMW ocular damage rabbit model using a gyrotron as the wave source and clarified the degree of ocular damage caused by $162 \mathrm{GHz}$ exposure for $6 \mathrm{~min}$, the clinical course of ocular damage, and the damage healing process. Although there were slight differences in the degree of ocular damage found in this study compared to our previous studies, there was no fundamental difference from previous reports of MMW induced ocular damage [5,7]. The ocular damage dose (DD) power densities at $162 \mathrm{GHz}$ MMW with probabilities of 10,50 , and $90 \%$ obtained by probit analysis were 173,252 , and $368 \mathrm{~mW} /$ $\mathrm{cm}^{2}$, respectively. 
Acknowledgments The authors are indebted to Ms. Megumi Koutou, Ms. Aki Hada, Dr. Tsutomu Okuno, Dr. Kensuke Sasaki, Dr. Soichi Watanabe, Mr. Yoshihisa Ishiba, and Ms. Mari Seto for their technical assistance, and to Mr. David Price for English proofreading.

Funding information This work was supported by Ministry of Internal Affairs and Communications, Grant Number JPMI10001, Japan.

\section{Compliance with Ethical Standards}

The animals were managed in accordance with practices and experimental protocols reviewed and approved by the Animal Research Committee, University of Fukui. All animal experiments were conducted in accordance with the animal experimentation guidelines of Kanazawa Medical University (Kahoku, Japan), University of Fukui (Fukui, Japan), and the ARVO Statement for the Use of Animals in Ophthalmic and Vision Research (ARVO, 2016) [13].

Conflict of Interest The authors declare that they have no conflict of interest.

Open Access This article is licensed under a Creative Commons Attribution 4.0 International License, which permits use, sharing, adaptation, distribution and reproduction in any medium or format, as long as you give appropriate credit to the original author(s) and the source, provide a link to the Creative Commons licence, and indicate if changes were made. The images or other third party material in this article are included in the article's Creative Commons licence, unless indicated otherwise in a credit line to the material. If material is not included in the article's Creative Commons licence and your intended use is not permitted by statutory regulation or exceeds the permitted use, you will need to obtain permission directly from the copyright holder. To view a copy of this licence, visit http://creativecommons.org/licenses/by/4.0/.

\section{References}

1. International Commission on Non-Ionizing Radiation Protection Guidelines for limiting exposure to time varying electric, magnetic and electromagnetic fields (up to $300 \mathrm{GHz}$ ), Health Phys, 74, 494-522 (1998)

2. S. W. Rosenthal, L. Birenbaum, I. T. Kaplan, W. Metlay, W. Z. Snyder and M. M. Zaret, Effects of 35 and $107 \mathrm{GHz}$ CW Microwaves on the rabbit eye, in Biological effects of electromagnetic waves. Selected Papers of the USNC/URSI Annual Meeting, Boulder, Colorado, October 1975. Rockville: US Department of Health, Education, and Welfare (HEW Publication (FDA) 77-8010, 1976), pp. 110-128

3. H. A. Kues, S. A. D'Anna, R. Osiander, W. R. Green and J. C. Monahan, Absence of ocular effects after either single or repeated exposure to $10 \mathrm{~mW} / \mathrm{cm}^{2}$ from a $60 \mathrm{GHz} \mathrm{CW}$ source, Bioelectromagnetics, 20, 463473 (1999)

4. S. Chalfin, J. A. D'Andrea, P. D. Comeau, M. E. Belt and D. J. Hatcher, Millimeter wave absorption in the nonhuman primate eye at $35 \mathrm{GHz}$ and $94 \mathrm{GHz}$, Health Phys, 83, 83-90 (2002)

5. M. Kojima, M. Hanazawa, Y. Yamashiro, H. Sasaki, S. Watanabe, M. Taki, Y. Suzuki, A. Hirata, Y. Kamimura and K. Sasaki, Acute ocular injuries caused by $60-$ Ghz millimeter-wave exposure, Health Phys, 97, 212-218 (2009)

6. M. Kojima, Y. Suzuki, C.-Y. Tsai, K. Sasaki, K. Wake, S. Watanabe, M. Taki, Y. Kamimura, A. Hirata, K. Sasaki and H. Sasaki, Characteristics of ocular temperature elevations after exposure to quasi- and millimeter waves (18-40 GHz), J Infrared Milli Terahz Waves, 36, 390-399 (2015)

7. M. Kojima, Y. Suzuki, K. Sasaki, M. Taki, K. Wake, S. Watanabe, M. Mizuno, T. Tasaki and H. Sasaki, Ocular Effects of Exposure to 40, 75, and 95 GHz Millimeter Waves, J Infrared Milli Terahz Waves, 39, 912-925 (2018)

8. M. Kojima, C.-Y. Tsai, Y. Suzuki, K. Sasaki, T. Tasaki, M. Taki, S. Watanabe and H. Sasaki, Ocular Response to Millimeter Wave Exposure Under Different Levels of Humidity, J Infrared Milli Terahz Waves, 40, 574-584 (2019)

9. C. M. Armstrong, The truth about terahertz, IEEE Spectrum, 49, 36-41 (2012)

10. A. Kasugai, K. Sakamoto, K. Takahashi, K. Kajiwara and N. Kobayashi, Steady-state operation of 170 GHz-1 MW gyrotron for ITAB, Nuclear Fusion, 48, 054009 (2008) 
11. Y. Tatematsu, Y. Yamaguchi, R. Ichioka, M. Kotera, T. Saito and T. Idehara, Development of the Multifrequency Gyrotron FU CW GV with Gaussian Beam Output, J Infrared Milli Terahz Waves, 36, 697-708 (2015)

12. K. Harima, Numerical Simulation of Far-Field Gain Determination at Reduced Distances Using Phase Center, IEICE Transactions on Communications, E97.B, 2001-2010 (2014)

13. ARVO Statement for the Use of Animals in Ophthalmic and Vision Research. Available from: https://www. arvo.org/About/policies/statement-for-the-use-of-animals-in-ophthalmic-and-vision-research/. Assessed 2 December, 2019

14. T. Tasaki, M. Kojima, Y. Suzuki, Y. Tatematsu and H. Sasaki, Creating a Stable Short-term Housing Environment for Rabbits in a Cargo Van, J Am Assoc Lab Anim Sci, 58, 456-461 (2019)

15. C. I. Bliss, The Method of Probits, Science, 79, 38-39 (1934)

16. R Core Team (2018). R: A language and environment for statistical computing. R Foundation for Statistical Computing, Vienna. Available from: https://www.R-project.org/. Assessed 2 December, 2019

17. K. Sasaki, T. Sakai, T. Nagaoka, K. Wake, S. Watanabe, M. Kojima, N. Hasanova, H. Sasaki, K. Sasaki, Y. Suzuki, M. Taki, Y. Kamimura, A. Hirata and H. Shirai, Dosimetry Using a Localized Exposure System in the Millimeter-Wave Band for in vivo Studies on Ocular Effects, IEEE Transactions on Microwave Theory and Techniques, 62, 1554-1564 (2014)

18. V. Aspinall and M. Cappello, Small exotic mammals, in Introduction to Veterinary Anatomy and Physiology Textbook 3rd edn (Butterworth-Heinemann, Oxford, 2015), pp. 166

Publisher's Note Springer Nature remains neutral with regard to jurisdictional claims in published maps and institutional affiliations.

\section{Affiliations}

\section{Masami Kojima $^{1,2,3} \cdot$ Yukihisa Suzuki $^{4} \cdot$ Takafumi Tasaki $^{5,6} \cdot$ Yoshinori Tatematsu $^{7}$. Maya Mizuno ${ }^{8}$ - Masafumi Fukunari ${ }^{7} \cdot$ Hiroshi Sasaki $^{1,2}$}

1 Division of Vision Research for Environmental Health, Medical Research Institute, Kanazawa Medical University, Kahoku, Japan

2 Department of Ophthalmology, Kanazawa Medical University, Kahoku, Japan

3 Medical Sciences, School of Nursing, Kanazawa Medical University, Kahoku, Japan

4 Department of Electrical Engineering and Computer Science, Graduate School of Systems Design, Tokyo Metropolitan University, Tokyo, Japan

5 Division of Protein Regulation Research, Medical Research Institute, Kanazawa Medical University, Kahoku, Japan

6 Department of Medical Zoology, Kanazawa Medical University, Kahoku, Japan

7 Research Center for Development of Far-Infrared Region, University of Fukui, Fukui, Japan

8 National Institute of Information and Communications Technology, Tokyo, Japan 